\title{
THE IMPACT OF VARIOUS PHYSIOTHERAPY PROGRAMS ON STATIC AND DYNAMIC BALANCE FOR PATIENTS AFTER STROKE
}

\author{
Dalia Mickevičienė, Erika Masiulytė, Neringa Švedaitė \\ Lithuanian Sports University, Kaunas, Lithuania
}

\begin{abstract}
Background. The imbalance in many scientific sources is described as a major disorder in patients with a history of stroke. Often, the importance or disruption of torso control is also indicated on balance impairments. We believe that both physiotherapy programs will have impact on balance however better results on balance tests will be in group which balance training composed of unstable planes. Research aim was to measure impact of various physiotherapy programs on static and dynamic balance for patients after stroke.

Methods. To evaluate changes of static and dynamic balance we used: Berg balance test, Tinetti test, PASS test, dynamic gait index, trunk impairment scale, special STREAM movements test for patients after stroke, Mini balance evaluation test for patients after stroke. Training methods of balance were for two different physiotherapy programs: intervention group (basic physiotherapy program with exercises on unstable planes) and control group (basic physiotherapy program with gait training elements).

Results. When evaluating balance, results in both groups were almost equal, which shows that balance was disturbed and there was a huge risk of falls, moreover all patients walked with aid measures. At the beginning of rehabilitation, the average of all balance evaluating tests in both groups scored 16.92 and 15.05. All results were statistically significant $(p<.05)$. At the end of research, variations between all test results were defined statistically significant.

Repeatedly evaluating results of both groups at the end of rehabilitation, all test averages were improved: at the beginning, the intervention group average score was 16.92 and after rehabilitation, it was 34.79 . For control group results, the average score of all tests was 15.05 and at the end it was 31.90. Summing up, it was established that in both groups balance in rehabilitation period improved.

Conclusions. Physiotherapy programs based on exercises on unstable planes or gait training elements improve balance for patients after stroke. Many studies have confirmed the hypothesis of our study that using unstable planes or walking training methods improve balance, but for a statistically significant change in improving the balance, it will be necessary to use unstable planes.
\end{abstract}

Keywords: physical therapy, stroke, balance, static, dynamic.

\section{INTRODUCTION}

$\mathrm{T}$ The imbalance in scientific literature is described as a major disorder in patients with a history of stroke. Often, the importance or disruption of torso control is also indicated on balance impairments (Heon \& Park, 2017).

According to the results of the study, after stroke, only one of five patients has lived more than 15 years, and lives an inferior life because each year, cognitive and functional abilities are deteriorating, which results in a person becoming less autonomous and more dependent on the surroundings (Crichton, Benjamin, McKevitt, Rudd, \& Wolfe, 2016).

Researchers say that torso muscle building and stabilization exercises should be included in the program not only to improve torstile control, 
but also as an effective method for balancing or walking (Gadhvi, Diwan, \& Vyas, 2016).

In a study done by Heon and Park (2017), the findings and results show that torso muscles are very closely related to balance and imbalance disorder, they are included in selective movements to control the centre of gravity while we are standing, during weight bearing and maintaining static and dynamic posture (Heon \& Park, 2017).

The findings of other studies have suggested that balance can be improved in the course of walking and exercise in which body weight bearing is required (Duijnhoven et al., 2016). According to scientists, walking improves along with balance (Yelnik et al., 2008).

\section{METHODS}

A total of 24 patients were included in the study, 19 of which were women and 5 men that were diagnosed with stroke. All patients participated voluntarily. Patients aged 50 to 80 years. The criteria for patient selection were the following:

1. Conscious, not having perceptions of disability - the result of mini mental test must be $>23$ points.

2. The patient could not be completely addicted, the Barthel self-determination index had to be $>62$ points.

3. The patient had to be able to move at least within the ward.

The total sample consisted of 24 patients who were randomly divided into two groups: 19 females and 5 males. Group 1: 9 females and 3 males. Group 2: 10 females and 2 males.

The subjects were tested twice: before and after a 7-week course of physiotherapy procedures.

Physical therapy was applied five times a week. The duration of one procedure was 40-45 minutes. Both groups included the same physical therapy structure: introduction, primary, and final, parts and both programs included muscle strengthening, balance training, and general body endurance exercises, but they were applied differently. Exercise of the research group is performed using only unstable surfaces - platforms, mats, balls, and the control group exercises were performed only by incorporating the elements of walking. The study was carried out at a department of rehabilitation in a hospital.

Berg Balance Scale was used for the assessment of static and dynamic balance. Berg
Balance Scale is one of the most popular scales that measure functional mobility and balance. It specializes in elderly people and is dedicated to assessing the human potential for safe daily tasks. Tasks vary in complexity: from a sitting position to standing on one leg. This test consists of 14 different tasks, each of which is scored in grades from 0 to 4 points. Score value is as follows: 0 points - a person cannot perform a task, balance is disturbed; 1 point - a person performs a task but needs help; 2 points - performs the task, but only after a few trials, uses minimal help; 3 points carries out the task independently, with minimal use of his/her body help; 4 points - ability to complete the task.

The maximum score is 56 . Assessment of the test results: $0-20$ points - the risk of falling is high, 21-40 points - the average risk of falling and 41-56 points $-\mathrm{a}$ low risk of falling. Berg and his co-authors found that, after collecting less than 45 points, there is an increased risk of falling.

Balance Assessment Systems Test. This test was developed by measuring 4 out of 6 different balance control systems and helping to tailor rehabilitation methods. This test is recommended by StrokeEDGE as a reliable test to measure poststroke changes in patient balance. The test evaluates the dynamic and static balance. The test consists of 14 different tasks, each of them is scored from 0 to 2. Score value: 0 points - cannot perform the task; 1 point - performs a task with help; 2 points performs the task without help.

The maximum score is 28 . Assessment of the test results: $0-15$ points - balance is disturbed, 15-25 points - balance is average, $25-32$ points balance is good.

The Dynamic Gait Index helps to assess patients' ability to perform tasks while walking, as well as changing the performance environment, the main assessment aspect is the risk of falling. The specialty of this index is the assessment of the quality of walking and balance of elderly patients, as well as patients who have experienced stroke. The assessment consists of dealing with tasks during walking and stepping up. The test consists of 8 tasks, each of them is scored from 0 to 3 points. Score value: 0 points - cannot perform the task; 1 point - ability to perform tasks inaccurately; 2 points - ability to perform tasks with auxiliary means; 3 points - perform the task.

Maximum score is 24. Assessment of test results: $0-19$ points - high risk of falling; $20-22$ 
points - average risk of falling; $22-24$ points there is no risk of falling.

Postural Scale for Stroke is one of the most popular scales for assessing the balance of stroke patients, regardless of torsion control abilities. This test is based on three main objectives: assessing the patient's ability to remain in a stable position, assessing balance in situations of change, and assessing skills when tasks are gradually getting harder. The test consists of two parts (tasks without changing the position and tasks by changing the position). A total of 12 different tasks, each of them is rated from 0 to 3 points. Score value: 0 points totally unbalanced, torso control impaired; 1 point - the aid used to maintain balance; 2 points the aid is not used, balance is maintained for a short time; 3 points - perfectly maintains balance.

The maximum score is 36 . Evaluation of the test results: $0-15$ points - balance and torso control are disturbed, 15-21 points - holds the static balance, but the movement needs help, 21-36 points - the balance and torso control are not distorted.

Mathematical Statistics. Statistical data analysis was performed using Microsoft ${ }^{\circledR}$ Excel 2010 and SPSS 23.0 programs. The sample sizes were compared with Wilcoxon and Mann-Whitney tests. Statistically significant differences were recorded at $p<.05$.

\section{RESULTS}

When evaluating both groups balance at the beginning of the study, results in both groups were almost equal, which shows that balance was disturbed and there was a huge risk of fall, moreover all patients walked using aids. At the beginning of rehabilitation, the average score of all balance evaluating tests in both groups was 16.92 and 15.05. In the course of the study, all results were statistically significant $(p<.05)$.

At the end of research, variation between all test results was defined as statistically significant. Repeatedly evaluating both groups at the end of rehabilitation, average results of both groups in all tests were improved: in the intervention group, the average at the beginning was 16.92 and after rehabilitation it was 34.79. The average of the control group results in all tests was 15.05 and at the end it was 31.90. Summing up, all results established show that balance in both groups in the rehabilitation period statistically significantly $(p<.05)$ improved.

In the research group, after the physiotherapy Berg test average score was $47.66 \pm 3.03$ points (Figure 1). In the control group, the average score was $40.67 \pm 1.83$ points. In both groups there was a statistically significant improvement $(p<.05)$.

In the evaluation of the balance changes after physical therapy with MiniBest balance test, the average score of the research group was $28.08 \pm$ 1.98 points. In the control group, the average score was $25.67 \pm 2.15$ points (Figure 2 ).

At the end of the study, better scores of the balance test scale of the research group could be explained by the fact that balancing training had greater efficiency with static and dynamic balance training using unstable planes.

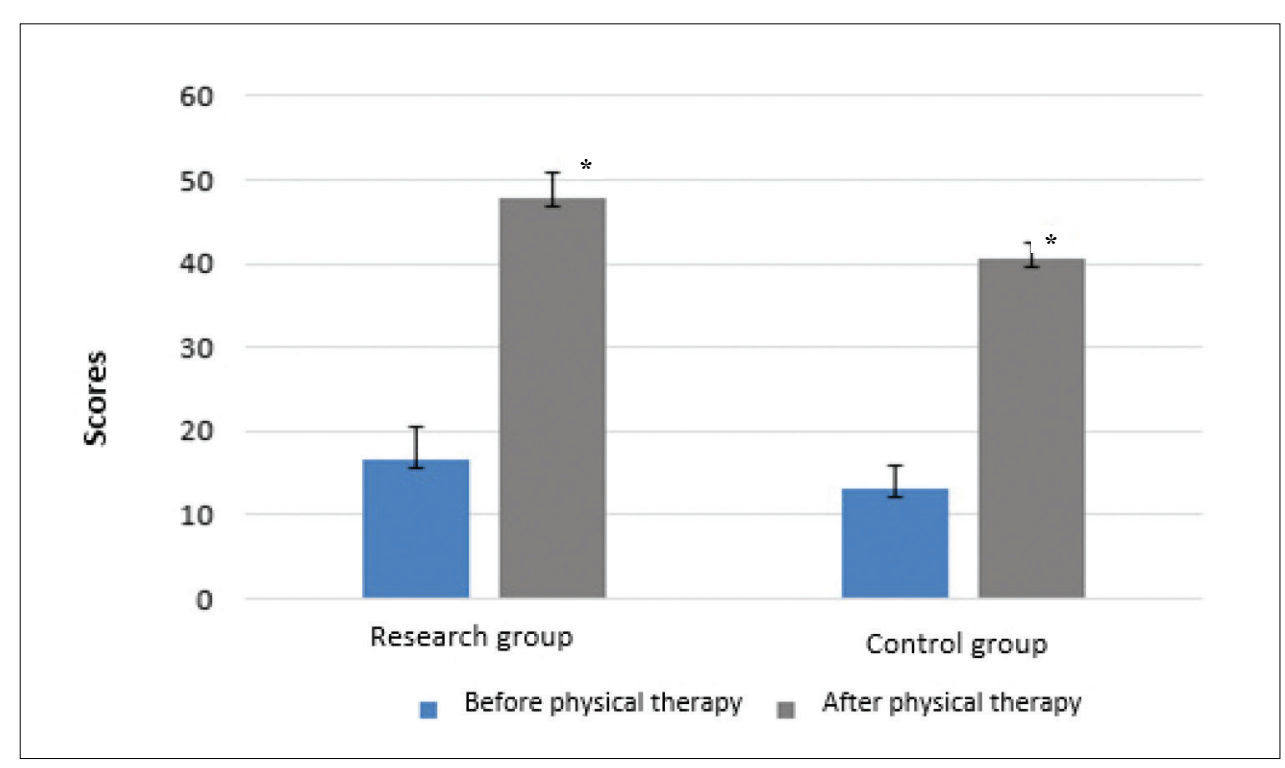

Figure 1. Berg Balance Scale value in Group 1 and Group 2 before and after physical therapy
Note. ${ }^{*} p<.002$, comparing the results in different groups before and after physical therapy. 
Figure 2. MiniBest test value in Group I and Group II before and after physical therapy

Note. ${ }^{*} p<.002$, comparing the results in different groups before and after physical therapy.

Figure 3. The Dynamic Gait Index value in Group 1 and Group 2 before and after physical therapy

Note. ${ }^{*} p<.002$, comparing the results before and after physical therapy in different groups.

Figure 4. Postural Assessment Scale for Stroke value in Group 1 and Group 2 before and after physical therapy

Note. ${ }^{*} p<.002$, comparing the results in different groups before and after physical therapy.
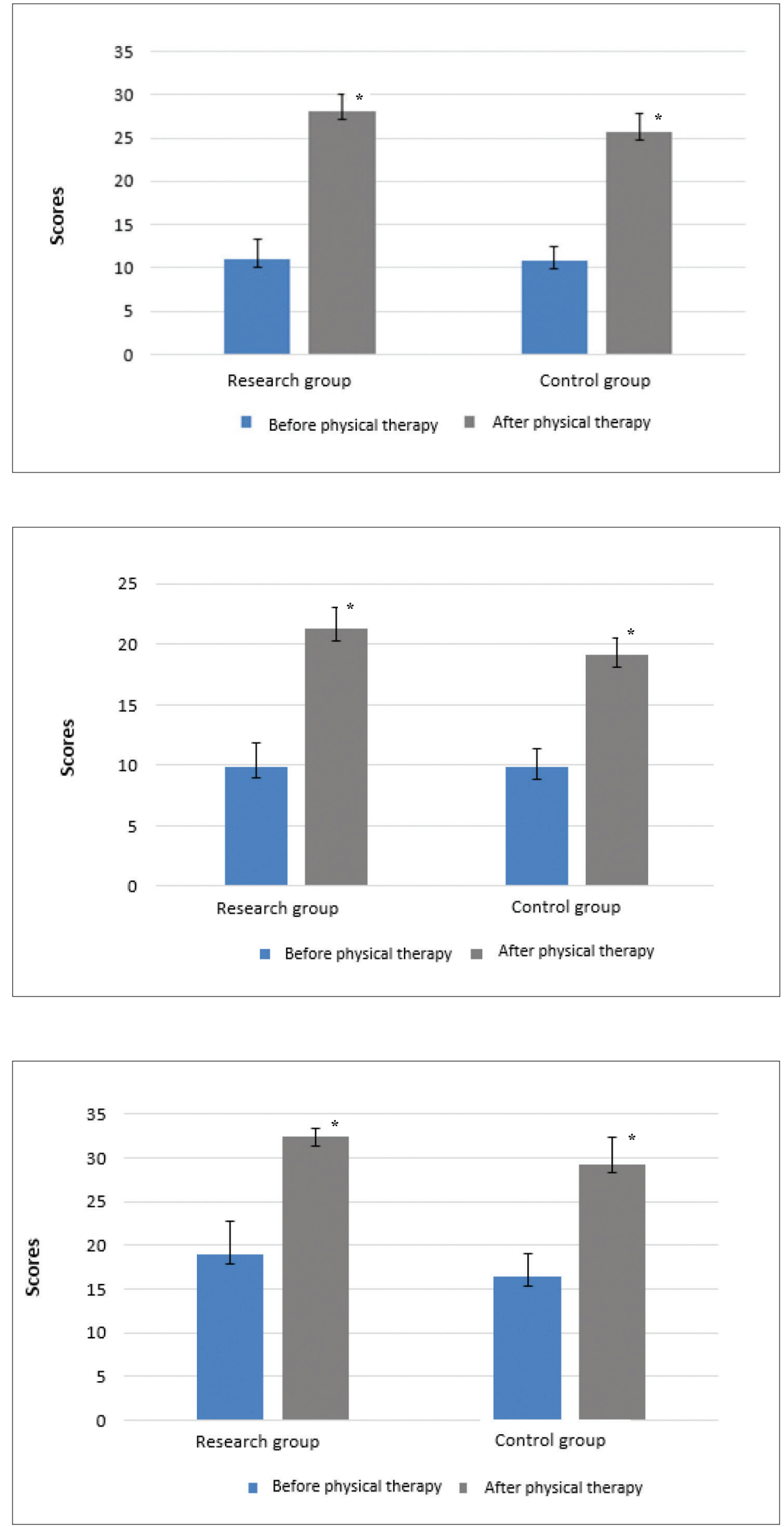
As shown in Figure 3, the mean of the Dynamic gait index results of the research and control groups at the end of the study showed a statistically significant improvement $(p<.05)$. The average score of the research group was $21.25 \pm 1.76$ points, and the average score of the control group was $19.17 \pm 1.40$ points.

In the research group, better scores of the balance test scale of the research group could be explained by the fact that balancing training had greater efficiency with static and dynamic balance training using unstable planes.

In the analysis of the data, the results of the two groups at the beginning of rehabilitation were initially different: the average score of the results of the research group was $18.92 \pm 3.75$ points; the average score of the control group results was 16.42 \pm 2.68 points. At the end of the study, the results of both groups were better than those at the beginning, the change in results was statistically significant ( $p$ $<.05$ ). The average score of the research group was $32.42 \pm 0.90$ points, the average score of the control group was $29.25 \pm 3.05$ points (Figure 4 ).

\section{DISCUSSION}

After analysing the data, we can see that after all physical therapy programs we applied, the results of all tests we did were improved, and their change was statistically significant $(p<.05)$. Only by comparing the results of the change between the groups, we can conclude that the first physiotherapy program that used the unstable planes to train achieved better results. Meanwhile, the second study group, whose balance was trained by walking, had worse results.

During the procedure, the control group performed exercises involving walking training elements. The results of our study showed that the walking elements had a positive effect on the improvement of balance. A similar study was conducted by Karbauskaite and co-authors (2017). The authors tracked changes in their study using a specialized walk-through program. During the study, the research group received a complex physical therapy program along with a specialized walk-through program, and the control group received a complex physical therapy program. In this study, a Berg balance scale and a dynamic gate index were used. The results obtained in both groups showed statistical significance $(p<.05)$, but the results of the research group had a higher statistically significant difference compared to the control group. The tests used in the study were chosen the same as we did. Analysing the results of the test, results at the end of the study were different from the results obtained by us. The average Berg balance scale was $51.82 \pm 3.6$ points, and our average score was $40.66 \pm 1.83$ points. The results of the dynamic gate index in the study were $21.27 \pm 2.8$ points, and the average score we obtained was $19.17 \pm 1.40$ points.

Kong and co-authors (2015) conducted a study to analyse the effects of different planes on the balance of patients with a history of stroke. During the study, the subjects were randomly divided into four groups, in which different surfaces were used for balance improvement. The tests that the authors chose were not the same as ours. The results of the study showed that changes in the results of all groups were statistically significant, but the third group, which used with physical therapy program with different unstable planes, had the highest statistical significance in assessing changes in balance than in the other three groups.

Yoo, along with co-authors (2014), conducted a study that assessed balance training using unstable platforms and core stabilization exercises. The Berg balance scale was used to assess the effectiveness of the study. The results of the study indicate that exercises using unstable planes have statistically significant results and are recommended as a good method for developing balance in patients with a history of stroke. The results obtained were similar to the results of our study - the average score for the assessed results among the groups was 5.69 points, and in our study it was 4.95 points.

Park, along with co-authors (2015), conducted a study in which balance was trained through walking tasks. The tests used in the study were not the same as we selected, but the results of the study indicate that balance improves because of walking training.

Bang and co-authors (2016) conducted a study in which balance was improving with walking exercises. Statistically significant differences were observed in the balance between the two groups (for the first group balancing exercise was walking tasks and the virtual game - Nintendo; the second group was subjected to a program based 
on special walking exercises and walking on the running track). When comparing changes in the groups, statistically significant changes were in the first group, which sustained from balance training exercises and virtual play.

Our research hypothesis was proven: the results of all tests used in the study between groups were statistically significant $(p<.05)$, so we can say that in patients who suffered from a stroke during rehabilitation, using a physical therapy program that involves exercises with unstable planes is more effective in balance training than a physical therapy program, including walking training elements. In addition, both physiotherapy programs had a positive effect on balance.

\section{CONCLUSIONS}

1. During physical therapy, the dynamic and static balance of both groups were statistically significantly improved.

2. Comparing the results between the groups, a statistically significant change was seen in the research group, the balance improving program of which consisted of a physical therapy program using unstable planes.

3. In both groups, subjects at the beginning of rehabilitation started walking with aids, and the results of the evaluated tests indicated that balance was disturbed, and at the end of the rehabilitation the subjects walked without the aids.

4. Balance training using unstable planes greatly improves the balance of patients with a history of stroke.

\section{REFERENCES}

Bang, Y. S., Son, K. H., \& Kim, H. J. (2016). Effects of virtual reality training using Nintendo Wii and treadmill walking exercise on balance and walking for stroke patients. The Journal of Physical Therapy Science, 26(2) 3112-3115. https://dx.doi.org/10.1589\%2Fjpts.26.271

Crichton, S. L., Benjamin, D. B., McKevitt, C., Rudd, G. A., \& Wolfe, C. D. A. (2016). Patient outcomes up to 15 years after stroke: survival, disability, quality of life, cognition and mental health. Cerebrovascular Disease, 87, 1091-1098.

Duijnhoven, H. J. R., Heeren, A., Peters, M. A. M., Veerbeek, J. M., Kwakkel, G., Geurts, A. C. H., \& Weerdesteyn, V. (2016). Effects of exercise therapy on balance capacity in chronic stroke. Stroke, 47, 26032610. doi: 10.1161/ STROKEAHA.116.013839

Gadhvi, J. B., Diwan, J. S., \& Vyas, N. J. (2016). Additional effect of trunk stabilization exercises on gait and balance in chronic stroke patients: An experimental study. International Journal of Therapies and Rehabilitation Research, 5(4), 33-38. https://doi. org/10.1002/jor.1100080310

Heon, S., \& Park, D. S. (2017). The effects of trunk exercise on mobility, balance and trunk control of stroke patients. Journal of the Korean Society of Physical Medicine, 12(1), 25-33.

Karbauskaitè, I., Mockevičienè, D., Norkienè, S., Razbadauskas, A., \& Sąlyga, J. (2017). Kineziterapijos, taikant specializuotą ejjimo programą, poveikis asmenims po išeminio galvos smegenų insulto. Reabilitacija, 27(6), 169-172.

Park, E. C., \& Hwangbo, G. (2015). The effects of action observation gait training on the static balance and walking ability of stroke patients. Journal of Physical Therapy Science, 27(2). https://dx.doi. org/10.1589\%2Fjpts.27.341

Yelnik, A. P., Breton, F. L., Colle, M. F., Bonan, I. V., Hugeron, C., Egal, ... Vicaut, E. (2008). Rehabilitation of balance after stroke with multisensorial training: A single-blind randomized controlled study. Neurorehabilitation and Neural Repair, 22(5), 468-476. doi: 10.5014/ajot.2015.011965

Yoo, J., Jeong, J., \& Lee, W. (2014). The effect of trunk stabilization exercise using a unstable surface on the abdominal muscle structure and balance of stroke patients. Journal of Physical Therapy Science, 26(6). https://doi.org/10.1177\%2F0269215515609414
Corresponding author Dalia Mickevičienè Institute of Sports Science and Innovations Lithuanian Sports University

Sporto str. 6, Kaunas LT-44221

Lithuania

Tel. +37060073021

Fax +370 37204515

Email dalia.mickeviciene@1su.1t 